\title{
L'effet produit sur les ondes solitaires par le frottement à la paroi
}

\author{
Arthur I. IPPEN \\ PROFESSEUR D'HYDRAULIQUE \\ Massachusets Institute of Technology
}

ET

English text, p. 401

\author{
GERshon KULIN \\ INGÉNIEUR HYDRAULICIEN \\ National Bureau of Standards
}

\begin{abstract}
L'article concerne les résultats des recherches expérimentales conduites au Laboratoire d'Hydrodynamique du Massachusetts Institute of Technology sur l'amortissement de l'amplitude des ondes solitaires. Les essais étaient effectués dans un canal en lucite de $9,75 \mathrm{~m}$ de longueur et $42 \mathrm{~cm}$ de largeur, la profondeur initiale de l'eau variant de $6 \dot{\alpha} 12 \mathrm{~cm}$ environ. Pour quelques-uns de ces essais le fond du bassin avait été rendu rugueux artificiellement par du gravier de grosseur de grain uniforme. Les amor-
\end{abstract}

\begin{abstract}
tissements ainsi observés sont comparés avec les théories existantes.

Au cours des essais, nous avons également cherché à aborder le problème de l'amortissement par une mesure directe de la couche limite transitoire voisine du fond. Une sonde différentielle spéciale avait été imaginée pour mesurer les vitesses transitoires faibles; on trouvera dans ce qui suit l'exposé des premiers résultats obtenus.
\end{abstract}

\section{INTRODUCTION}

L'onde solitaire (fig. 1) comprend ordinairement une crête unique symétrique, ni précédée ni suivie par un creux. Une onde de ce genre se déplace sans modifier son profil, donné très exactement par l'équation de BoussinesQ [1] :

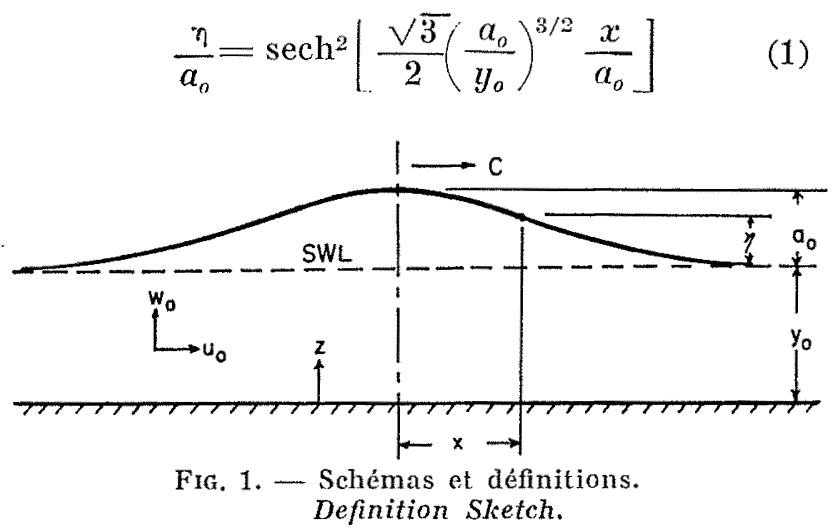

$\eta$ étant la surélévation au-dessus de la ligne d'eau originelle à une distance quelconque $x$ à partir de la crête, $a_{o}$ la valeur maxima de $\eta$, et $y_{o}$ la profondeur de l'eau au repos.
La célérité de cette intumescence est donnée par l'équation de Boussinese et par Rayleigh [2]:

$$
\frac{\mathrm{C}}{\sqrt{g y_{0}}}=\sqrt{1+\frac{a_{0}}{y_{0}}}
$$

où $\mathrm{C}$ désigne la célérité et $g$ l'accélération de la pesanteur. Ainsi donc, en première approximation, la célérité de l'onde solitaire peut être représentée par l'expression classique de la vitesse de propagation des perturbations infinitésimales en eau peu profonde :

$$
\mathrm{C}=\sqrt{g y_{o}}
$$

Les vitesses à l'intérieur de l'onde sont données par les équations McCowan [3], rendues non-dimensionnelles par MUNK [4]:

$$
\begin{aligned}
& \frac{u_{0}}{\mathrm{C}}=\mathrm{N} \frac{1+\cos \mathrm{M} z / y_{0} \times \cosh \mathrm{M} x / y_{0}}{\left(\cos \mathrm{M} z / y_{0}+\cosh \mathrm{M} x / y_{0}\right)^{2}} \\
& \frac{w_{o}}{\mathrm{C}}=\mathrm{N} \frac{\sin \mathrm{M} z / y_{0} \times \sin \mathrm{M} x / y_{0}}{\left(\cos \mathrm{M} z / y_{0}+\cosh \mathrm{M} x / y_{0}\right)^{2}}
\end{aligned}
$$


où $u_{o}$ et $w_{o}$ désignent respectivement la composante horizontale et la composante verticale de la vitesse de l'élément fluide à une hauteur $z$ à partir $\mathrm{du}$ fond. $\mathrm{M}$ et $\mathrm{N}$ sont sensiblement égaux à $\sqrt{3 a_{o} / y_{0}}$ et à $2 a_{0} / y_{o}$ respectivement. On voit que pour les ondes ayant une amplitude relative (par rapport à la profondeur) très faible, les vitesses internes sont à peu près entièrement horizontales et approximativement exprimées par :

$$
u_{o}=\mathrm{C} \frac{\eta}{y_{o}}
$$

la vitesse des particules fluides est alors indépendante de la profondeur.

Pour vérifier la validité des équations (1), $(2),(3 a)$ et $(3 b)$, on a procédé au Laboratoire d'Hydrodynamique du Massachusetts Institute of Technology à des essais en bassin de lucite, de $9,75 \mathrm{~m}$ de longueur et $42 \mathrm{~cm}$ de largeur [5]. Les profondeurs initiales s'échelonnaient entre 6 et $12 \mathrm{~cm}$.

Le volume par unité de largeur (en pieds) de l'onde solitaire peut être exprimé par :

$$
\frac{v}{a_{o}^{2}}=\frac{4}{\sqrt{3}} \frac{1}{\left(a_{o} / y_{0}\right)^{3 / 2}}
$$

et son énergie rapportée au pied $(0,30 \mathrm{~m})$ de largeur, par :

$$
\frac{\mathrm{E}}{\gamma a_{o}{ }^{3}}=\frac{2}{3}\left(\frac{4}{\sqrt{3}} \times \frac{1}{\left(a_{o} / y_{0}\right)^{3 / 2}}\right)=\frac{2}{3} \frac{v}{a_{o}{ }^{2}}
$$

$\gamma$ étant le poids spécifique de l'eau. Les équation (5) et (6) ont été obtenues avec un profil défini par l'équation (1) de BoussinesQ. L'équation (6) présuppose une onde d'amplitude extrêmement faible par rapport à la profondeur.

L'onde solitaire peut être considérée comme un cas limite de l'onde périodique en eau peu profonde. La ressemblance très accusée entre les ondes périodiques en profondeur décroissante et un «train d'ondes solitaires» rend particulièrement intéressantes les propriétés de l'onde solitaire en ce qui concerne l'étude du comportement d'une onde périodique au voisinage du point de déferlement.

\section{Amortissement sur fond lisse}

L'amortissement de l'onde solitaire peut être tout d'abord attribué à la perte d'énergie au sein d'une couche limite relativement mince qui se forme le long du fond et des parois latérales du bassin. En dehors de cette région fontière, l'écoulement conserve essentiellement l'aspect décrit par la théorie potentielle.

Le mouvement des particules fluides à l'intérieur d'une onde solitaire se fait seulement dans la direction du déplacement de l'onde, sans courant de retour. Une particule fluide primitivement au repos subit une accélération en direction du déplacement de l'onde au passage de la pente antérieure de l'onde. Elle atteint sa vitesse maxima au passage de la crête, après quoi elle ralentit et revient au repos quand la perturbation, qui est de longueur pratiquement finie, est passée en aval. Une particule fluide proche du fond chemine, sous l'action de l'onde, en ligne droite, tandis que la trajectoire d'une particule de la surface est sensiblement parabolique.

De considérations physiques concernant le mouvement des particules près des parois, on peut déduire l'existence d'une couche limite d'épaisseur croissant depuis le front jusqu'à l'arrière de l'onde, car cet arrière correspond aux extrémités des trajectoires des particules. Au passage de la pente arrière de l'onde, les particules de la couche limite subissent un gradient de contre-pression et la possibilité d'une séparation de la couche limite est à prendre en considération.

Il serait utile d'avoir un nombre de Reynolds caractérisant le mouvement interne de l'onde. Dans la théorie de la plaque plane en écoulement permanent, l'épaisseur de la couche limite dépend d'un nombre de Reynolds fonction de la vitesse constante dans la région d'écoulement à potentiel et de la longueur de l'écoulement le long de la plaque. On pourra utiliser un nombre de Reynolds similaire dans les problèmes d'ondes, à condition de faire abstraction de la nonpermanence du mouvement. Cela revient à ramener la question au cas des ondes très plates et très longues, restriction compatible avec le domaine auquel s'appliquent les équations $(2 a)$, (4) et (5).

Un nombre de Reynolds pour ondes solitaires, ayant une signification physique, peut donc être défini par :

$$
\mathrm{R}_{\iota}=\int \frac{u_{o} d \zeta}{\nu}
$$

$\zeta$ étant le déplacement horizontal des particules fluides, $u_{n}$ la vitesse d'écoulement à potentiel en dehors de la couche limite et $\vee$ le coefficient de viscosité cinématique.

Compte tenu de :

et

$$
\begin{aligned}
& u_{o}=\frac{d \zeta}{d t} \\
& \mathrm{C}=\frac{d x}{d t}>>u_{n}
\end{aligned}
$$

l'équation (7) devient :

$$
\mathrm{R}_{\mathrm{c}}=\frac{\mathrm{C}}{y y_{0}{ }^{2}} \int_{r}^{\infty} \eta^{2} d x
$$

Selon la théorie du cas des faibles amplitudes, 
la quantité sous l'intégrale est proportiomnelle à l'énergie de l'onde. En utilisant les équations (2a) et $(6)$ et après intégration sur toute la longueur de l'onde on obtient pour le nombre de Reynolds relatif de l'onde solitaire, c'est-à-dire pour le nombre de Reynolds de la plaque plane équivalente :

$$
R_{\varepsilon}=1,54 \frac{a_{\mathrm{o}} \sqrt{g a_{n}}}{y}
$$

Si Yon attribue à $v$ et à $g$ les valeurs moyennes respectives $1 / 100.000 \mathrm{ft}^{2} / \mathrm{sec}\left(1 / 93 \mathrm{~cm}^{2} / \mathrm{s}\right)$ el $32 \mathrm{ft} / \mathrm{sec}^{2}\left(9,80 \mathrm{~m} / \mathrm{s}^{2}\right)$, on $\mathrm{a}$ :

$$
\mathrm{R}_{\complement} \approx 870.000 a_{0}{ }^{3 / 2}
$$

$a_{o}$ étant exprimé en pieds.

Ce nombre de Reynolds, bien qu'approximatif, permet d'évaluer le régime d'écoulement à l'intérieur de la couche limite. Sa valeur la plus élevée, obtenue pendant l'étude expérimentale en question, était d'environ 120.000. Par comparaison avec les valeurs connues pour la plaque plane, on peut estimer que l'écoulement dans la couche limite est très largement à l'intérieur du domaine laminaire.

En se rapportant de nouveau à la théorie de la plaque en écoulement permanent, on pourra exprimer l'épaisseur d'une couche laminaire où règne une distribution parabolique des vilesses, au passage d'une onde solitaire, par :

$$
\delta_{x}=\frac{5, \overline{5} \zeta_{x}}{\sqrt{\mathrm{R}_{\zeta_{x}}}}
$$

L'indice inférieur $« x »$ correspond aux résultats cumulés depuis l'infini positif jusqu'à un point $x$ le long de l'onde. En première approximation, la longueur $\zeta$ du déplacement de la particule ne dépend pas de la hauteur; elle est égale au volume de l'intumescence divisé par la profondeur. Les équations (5) et (13) donnent alors pour l'épaisseur de la couche limite au passage de l'arrière de l'onde solitaire :

$$
\frac{\hat{o}_{n}=-\infty}{y_{0}}=\frac{12,7}{\sqrt{\mathrm{R}_{\mathrm{L}}}} \sqrt{\frac{a_{n}}{y_{0}}}
$$

et au passage de la crête :

$$
\partial_{x=0}=\frac{1}{\sqrt{2}} \partial_{x^{i}}=-\infty
$$

L'équation (14) ne tient pas compte du caractère non permanent du mouvement et n'envisage pas la possibilité d'un courant de retour au passage de l'extrémité arrière de l'onde. Des considérations physiques font penser à l'existence d'un tel courant, qui découle d'ailleurs du déve- loppement de Keulegan [6] concernant le frottement à la paroi. Néanmoins, l'équation (14) fournit une estimation directe et facile de l'épaisseur que doit avoir la couche limite sous une onde de faible amplitude.

En égalant la perte d'énergie due à l'amortissement visqueux dans la couche limite à la perte d'énergie correspondant à la réduction de l'amplitude, KEULEGAN [6] a obtenu pour l'affaiblissement de l'onde solitaire l'expression :

$$
\begin{aligned}
& \left(\frac{a_{n}}{y_{n}}\right)^{-1 / 4}-\left(\frac{a_{i}}{y_{0}}\right)^{-1 / 4} \\
& \quad=\frac{1}{12}\left(1+\frac{2 u_{n}}{\mathrm{~B}}\right) \sqrt{\frac{y}{g^{1 / 2} y_{0}^{3 / 2}}} \times \frac{s}{y_{0}}=\mathrm{K} \frac{s}{y_{0}}
\end{aligned}
$$

$B$ indique la largeur du chenal, $s$ la distance sur laquelle l'amplitude est atténuée depuis $a_{i}$ jusqu'à $a_{0}$. On voit que le coefficient d'affaiblissement est constant pour une profondeur donnée de l'eau.

La forme de l'équation (16) a été vérifiée expérimentalement [5]. On observait photographiquement chaque onde tout au long de son mouvement de va-et-vient par réflexion aux extrémités du bassin de 9,75 m. Pour ces expériences, et pour toutes les autres mentionnées dans le présent article, on engendrait l'onde solitaire en levant rapidement une vanne, de façon à libérer un faible volume d'eau retenue; celle-ci à son tour déplaçait doucement une paroi verticale sur une faible distance vers l'aval. A chacune des profondeurs, on observait plusieurs ondes d'amplitude initiale différente, jusqu'à complète extinction. On obtint ainsi les coefficients d'affaiblissement moyens suivants :

\begin{tabular}{c|c|c}
\hline Profond $^{\mathrm{r}}$ en $\mathrm{ft}$ & $\mathrm{K}$ de Keulegan & $\mathrm{K}$ expérimental \\
\hline $0.2(0,06 \mathrm{~m})$ & 0,000480 & 0,000372 \\
$0.3(0,09 \mathrm{~m})$ & 0,000379 & 0,000327 \\
$0.4(0,12 \mathrm{~m})$ & 0,000335 & 0,000318
\end{tabular}

On voit que le coefficient expérimental dépend moins de la profondeur que le coefficient théorique.

Dans le cas considéré, les différences entre valeurs théoriques et expérimentales peuvent être facilement prévues, parce que :

1. En théorie, pour des raisons de commodité, on se limite aux premières approximations pour la célérité de l'onde, pour l'énergie et pour les vitesses internes;

2. Le terme correctif $\left[1+\left(2 y_{0} / \mathrm{B}\right)\right]$, tenant compte de la paroi latérale, est très approximatif pour les ondes de grande et moyenne hauteur; 
3. Les réflexions successives qu'on doit créer quand on étudie l'amortissement dans un canal expérimental donnent lieu à une oscillation secondaire; il s'ensuit que l'onde ne se déplace jamais dans une eau parfaitement calme.

Par définition, une onde solitaire conserve son volume initial pendant qu'elle s'atténue, tandis que l'onde de la théorie potentielle perd de son volume à mesure que son amplitude diminue. Des essais [7] semblent montrer que cette variation de volume est concentrée aux extrémités de l'onde, mais que la partie centrale du profil d'une onde même fortement amortie est bien définie par la théorie potentielle (équation 1). Il a été constaté que cette différence entre volume théorique et volume réel influe de façon négligeable sur le processus de l'amortissement.

Dans le but d'étudier l'amortissement d'une onde solitaire sans recourir aux réflexions successives, l'étude a été faite des résultats fournis par des essais qui ne portaient que sur le premier passage de l'onde dans le canal après sa génération. Les résultats sont indiqués sur la figure 2. La distance qui séparait les deux pre-

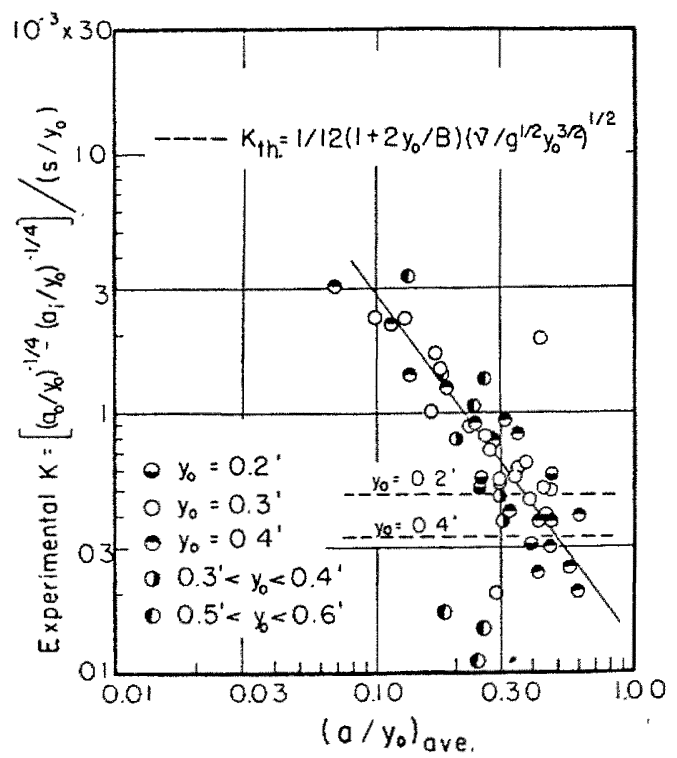

FIG. 2. - Coefficients expérimentaux d'amortissement correspondant au premier passage de l'onde.

Experimental first-pass Damping Coefficients.

mières stations d'observation étant de $3,65 \mathrm{~m}$ seulement, la diminution d'amplitude observée était faible; la dispersion des valeurs expérimentales était donc grande. Toutefois une tendance nette apparaît, montrant que le coefficient d'amortissement varie comme l'inverse de la hauteur de l'onde; on a donc été amené à porter les coefficients sur un diagramme en fonction du nombre de Reynolds $R_{\digamma}$ (fig. 3). La dispersion est accrue, mais on distingue quand même une certaine relation dont nous nous occuperons au paragraphe suivant.

Dans le domaine des $a_{o} / y_{o}$ faibles, où les hypo-

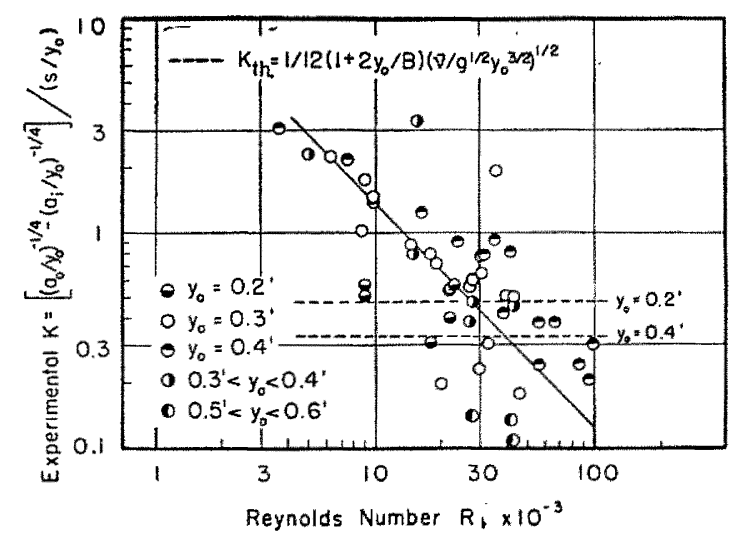

FIG. 3. - Coefficients d'amortissement en fonction du nombre de Reynolds (avant réflexion).

First-pass Damping Coefficients vs. Reynolds Number.

thèses simplificatrices de la théorie de KeuleGAN sont à peu près vérifiées, les coefficients d'amortissement semblent sur la figure 2 un peu plus élevés que ne le prévoit la théorie. On ignore la raison de l'écart, mais on peut expliquer la forte pente de la courbe expérimentale relativement à la ligne horizontale théorique en comparant les vitesses réelles à l'intérieur de l'onde aux vitesses théoriques données par l'équation (4).

\section{Amortissement sur fond rendu rugueux artificiellement}

L'équation de l'amortissement, citée au paragraphe précédent (éq. 16) et obtenue par KEvLEGAN [6], est limitée au cas d'une dissipation d'énergie à l'intérieur d'une couche laminaire transitoire sous des ondes solitaires passant dans des canaux lisses. Son domaine d'application est donc limité aux valeurs de $R_{c}$ (l'équivalent du nombre de Reynolds pour plaque plane), adopté ordinairement dans le cas de couches limites laminaires. Nous avons donc cherché, par un procédé d'application plus générale, à trouver une relation englobant les valeurs de l'amortissement obtenues pour des surfaces rugueuses et convenant au-delà du domaine de l'amortissement laminaire, à l'amortissement avec couche limite en régime de transition et en régime turbulent.

Si l'on définit, comme d'ordinaire, la résistance de surface instantanée en un point par le frottement unitaire :

$$
\tau=c_{f} \frac{\omega u_{s}^{2}}{2}
$$


on aura :

$\frac{d \mathrm{E}}{d t}=\int_{-\infty}^{+\infty} \tau u_{0} d x=\int_{-\infty}^{+\infty} c_{f} ? \frac{u_{0}^{3}}{2} d x$

Après substitution des équations (1) et (4) et remplacement de $c_{f}$ par un coefficient moyen $\mathrm{C}_{f}$ applicable à toute la longueur de l'onde, on pourra intégrer (18) et on obtiendra :

$$
\frac{d \mathrm{E}}{d t}=\frac{16}{15 \sqrt{3}} \rho \mathrm{C}_{f} g^{3 / 2} a_{o}^{5 / 2}
$$

Une seconde expression pour $d \mathrm{E} / d t$ est obtenue par différentiation de l'équation (6) :

$\frac{d \mathrm{E}}{d t}=\frac{d \mathrm{E}}{d a} \cdot \frac{d a}{d s} \mathrm{C}=\frac{4}{\sqrt{3}} \bullet g^{3 / 2} y_{o}^{2} a_{o}^{1 / 2} \frac{d a}{d s}$

Egalant (19) et (20) on pourra évaluer $\mathrm{C}_{f}$ pour des valeurs locales de $d a / d s$ et $a_{o} / y$ tirées de données expérimentales; il vient :

$$
\mathrm{C}_{f}=\frac{15}{4}\left(\frac{a_{0}}{y_{0}}\right)^{-2} \frac{d a}{d s}
$$

Remarquons cependant que cette expression générale n'est pas intégrable, à moins qu'on ne puisse exprimer $C_{f}$ comme une fonction de $R_{\xi}$ et d'une rugosité relative $e / a_{o}$ qu'on déterminera d'ordinaire expérimentalement. Dans le cas où $\mathrm{C}_{f}$ serait peu sensible aux changements subis par un de ces paramètres, c'est-à-dire aux fortes valeurs du nombre de Reynolds et aux grandes valeurs de $e / a_{o}$, l'équation (21) pourra être intégrée et donnera :

$$
\left(\frac{a_{0}}{y_{0}}\right)^{-1}-\left(\frac{a_{i}}{y_{0}}\right)^{-1}=\frac{4}{15} \mathrm{C}_{f} \frac{s}{y_{0}}
$$

Mais nous avons dit ci-dessus que les ondes ayant fait l'objet de cette étude n'étaient pas suffisamment grandes pour permettre de trouver la façon dont la résistance se comportera au-delà du domaine correspondant à la couche limite laminaire. Toutefois, comme on le verra par la suite, il est possible de calculer et d'analyser méthodiquement un certain effet de la rugosité.

Diverses séries d'essais d'amortissement ont été réalisées, le fond du bassin étant couvert d'une couche de gravier uniforme, mais dont on faisait varier le diamètre entre 1,7 et $5,9 \mathrm{~mm}$ tout au long des essais. La figure 4 reproduit sous forme de courbes de $a_{o} / y_{o}$ en fonction de $s / y_{o}$ les résultats de ces expériences avec fond rugueux. Toutes ces courbes ont été corrigées de façon approximative de l'effet de la résistance des parois latérales lisses à l'aide de l'équation (16). Pour permettre la comparaison, on a groupé les courbes par profondeur sur la figure $4 a$ et selon le paramètre de rugosité $e / y_{o}$ sur la figure $4 \mathrm{~b}$. A noter que, sur la figure $4 b$, les courbes de même valeur $e / y_{0}$ ont été décalées, quand il le fallait pour faciliter la comparaison, et amenées à une origine ayant le même rapport (amplitude initiale : profondeur), $a_{i} / y_{0}$. On remarque que, sauf pour les essais (9) et (2), l'amortissement est d'ordinaire plus rapide quand la profondeur est moindre. Cependant, en ce qui concerne l'essai (2), celui-ci a donné tout au long de cette étude des valeurs incompatibles avec les autres; il est très probablement erroné par suite de défauts d'enregistrement. Dans leur ensemble, les courbes révèlent de façon prédominante un amortissement visqueux, dont les intensités dépendent manifestement de la résistance de forme des particules constituant la rugosité.

Les effets de celle-ci ont été étudiés plus en détail; on portait les valeurs de $d a / d s$, déduites des courbes d'amortissement initiales en fonc-

Fig. 4. - Courbes d'amortissement sur fond rugueux. Roughened Bottom Attenuation Curves.

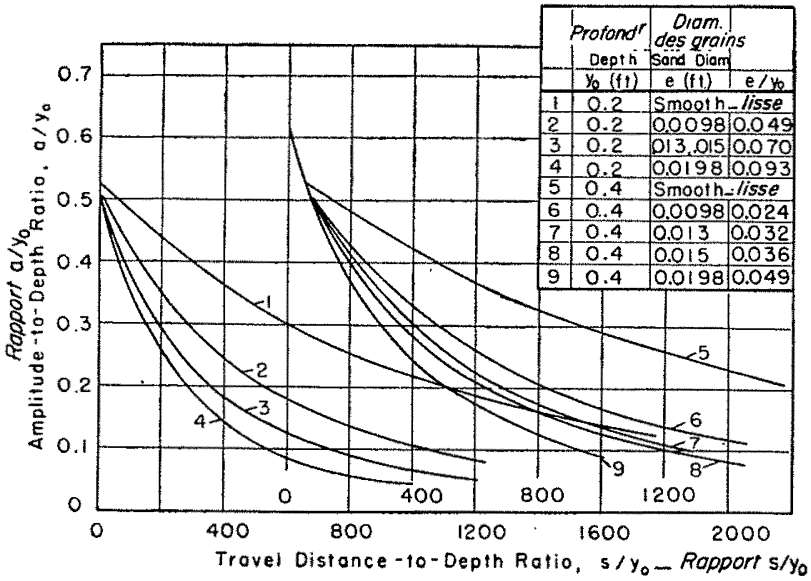

a) Courbes groupées par profondeur.

a) Curves grouped for equal Depth.

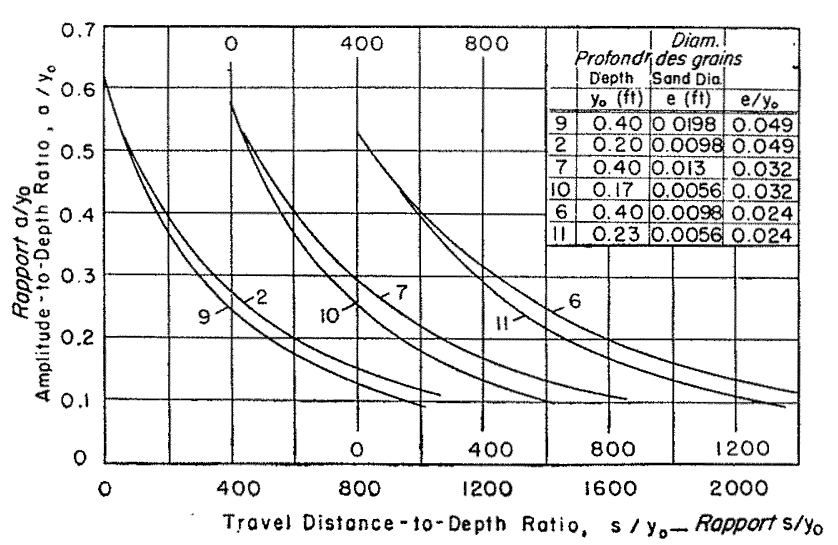

b) Courbes groupées par rugosité relative. b) Curves grouped for equal relative Roughness. 


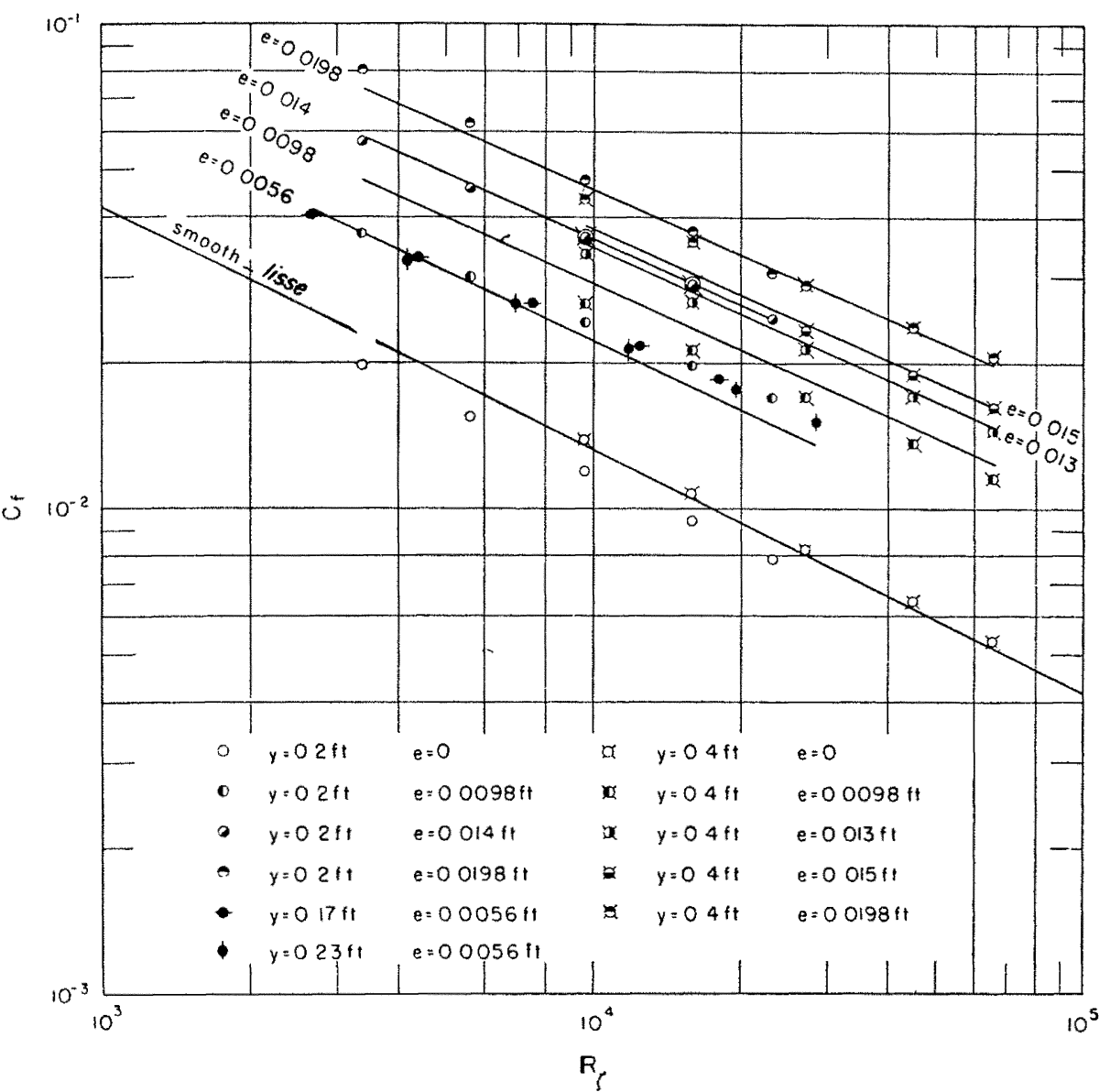

a) Coefficient de résistance en fonction du nombre de Reynolds. a) Plot of Resistance Coefficient versus Wave Reynolds Number.

b) Evaluation de la constante de l'équation (23 a).

b) Evaluation of Constant

in Equation $(23 a)$.

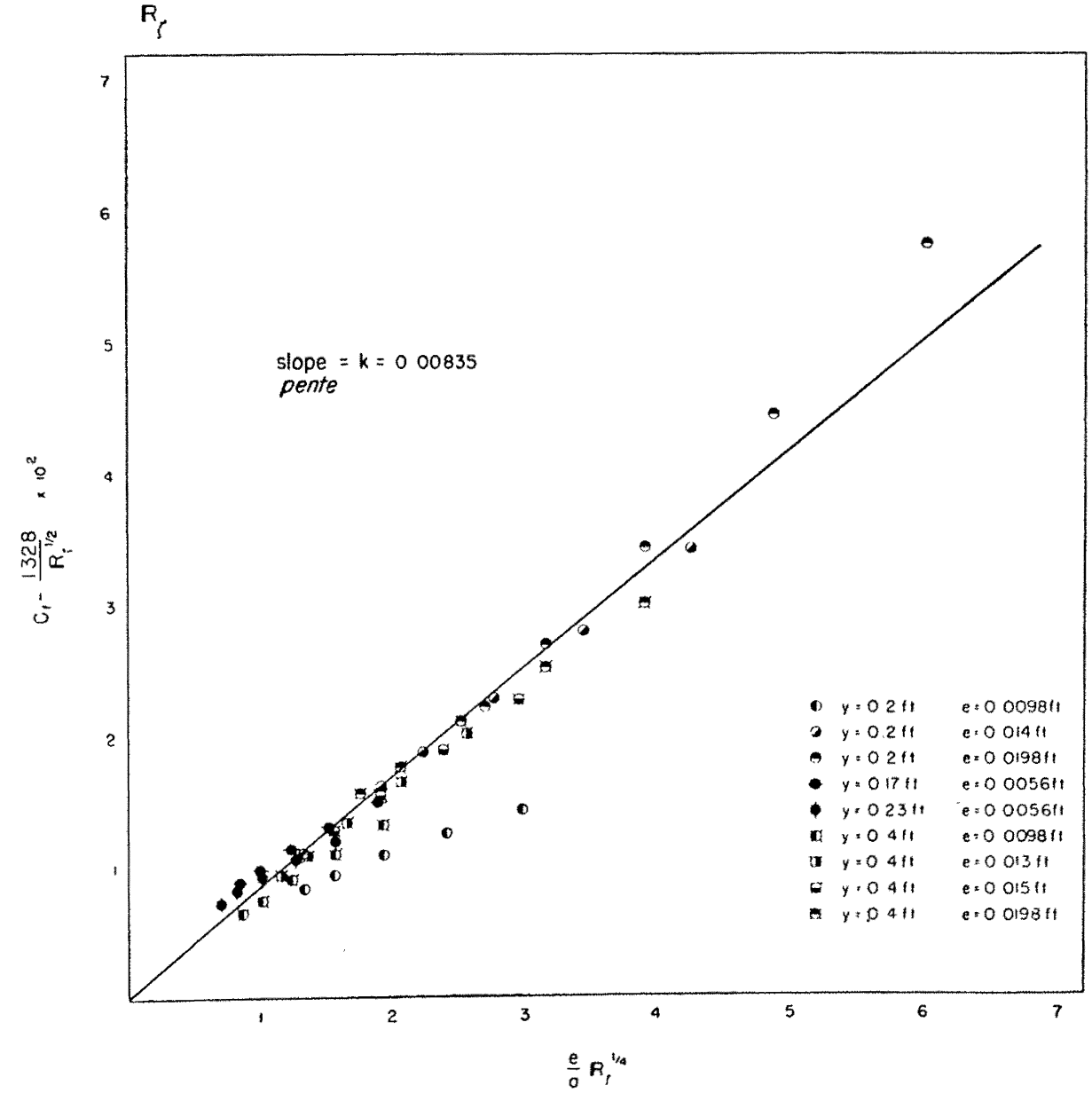

Frg. 5. - Coefficients de résistance globale pour fonds lisses et rugucux.

General Resistance Coefficients for smooth and roughened Bottom. 
tion de $a_{0} / y_{0}$, et on calculait les coefficients de résistance $\mathrm{C}_{f}$ d'après l'équation (21). Les résultast de cette étude sont donnés sur la figure $5 a$. $\mathrm{C}_{f}$ décroît généralement à mesure qu'augmente le nombre de Reynolds $R_{\xi}$, à la manière du coefficient de résistance pour surface lisse $\mathrm{C}_{f}$ défini par la formule de Blasius $\mathrm{C}_{f} \mathrm{~V}^{\prime} \mathrm{R}=1,328$ pour les couches limites laminaires sur plaques planes. Les valeurs pour le cas du canal lisse concordent à merveille avec la loi de Blasius et ajoutent considérablement à la confiance que mérite le nombre de Reynolds de l'onde $\mathrm{R}_{\llcorner}$, précédemment obtenu. A noter aussi que sa valeur est déterminée par la seule amplitude. Il a été d'autre part constaté que le décalage en direction verticale des valeurs de $C_{f}$ correspondant à des valeurs données de $R_{\zeta}$ était essentiellement régi par la rugosité absolue $e$. Les relations introduisant la notion d'une longueur de plaque équivalente ou de l'épaisseur de la couche limite laminaire n'ont pas été marquées de succès jusqu'ici, peut-être à cause des données trop peu nombreuses qu'on possédait. Il y a lieu de noter ici que les épaisseurs de la couche limite déduites des équations (14) et (15) sont en gros de l'ordre des diamètres des particules constituant la rugosité dans les expériences sur fond rendu rugueux. Il a cependant été possible de définir la rugosité relative comme étant $e / a_{o}$ et de déduire des observations une expression empirique pour $\mathrm{C}_{f}$ en fonction de $\mathrm{R}_{\mathcal{\zeta}}$ et de $e / a_{0}$ :

$$
\mathrm{C}_{f}=\frac{1,328}{\sqrt{\mathrm{R}_{\llcorner}}}+0,00835 \frac{e}{a_{\mathrm{o}}} \mathrm{R}_{\zeta}{ }^{1 / 4}
$$

ou encore :

$$
\mathrm{C}_{f}=\frac{1,328}{\sqrt{\overline{\mathrm{R}_{\varepsilon}}}}\left(1+0.0063 \frac{e}{a_{\mathrm{o}}} \mathrm{R}_{\varepsilon}^{3 / 4}\right)
$$

Sur cette dernière formule, on reconnaît facilement que la correction pour rugosité modifiant la constante de Blasius ne dépend que très peu de l'amplitude $a_{o}$; elle dépend surtout de la rugosité absolue, dans le domaine étudié. La valeur 0,00835 de cette constante a été obtenue d'après le graphique particulier de la figure $5 b$ où les indications de l'expérience sont particulièrement claires. Encore une fois, si l'on excepte l'essai précité avec $y_{o}=0,06 \mathrm{~m}$ et $e=0,0029 \mathrm{~m}$, les résultats concordaient assez bien avec les équations ci-dessus.

Il est évident que les ondes engendrées dans les bassins utilisés pendant les essais n'étaient pas de dimensions suffisantes pour créer des couches limites turbulentes et que les saillies formant la rugosité se trouvent principalement dans une région d'écoulement visqueux. Nous avons déjà dit que le nombre de Reynolds le plus grand obtenu pour ces ondes atteignait environ 120000 ; il devrait être notablement plus élevé pour créer un effet de turbulence dans la couche limite, malgré la présence d'un mouvement non permanent et d'un fond rendu rugueux; autant de facteurs susceptibles d'avancer le passage de l'écoulement laminaire à l'écoulement turbulent. On peut admettre qu'il faudraịt une onde d'amplitude initiale légèrement supérieure à $0,30 \mathrm{~m}$ pour déterminer un amortissement de type «rugueux» sur une portion notable du parcours expérimental. Cette amplitude exigerait une profondeur d'eau d'au moins $1.5 \mathrm{ft}(0,5 \mathrm{~m})$ et l'on pourrait s'attendre à une transition graduelle depuis l'amortissement du type exprimé par l'équation (16) à celui qu'exprime l'équation (17). De nouvelles recherches sont actuellement entreprises dans ce domaine.

Il est également évident qu'on peut introduire dans l'équation (21) le $\mathrm{C}_{f}$ défini par les équations (23) en vue de l'intégration et calculer ensuite les courbes d'amortissement laminaire pour des canaux lisses et pour des canaux rugueux. Pour des canaux lisses, le coefficient $\mathrm{C}_{f}$ étant celui de Blasius, on trouve après intégration l'équation de l'amortissement de Keulegan (16) avec le seul changement du coefficient numérique, passant de $1 / 12$ à $1 / 14$. Une pareille concordance des deux méthodes procure l'assurance d'une bonne interprétation physique du nombre de Reynolds Rc pour plaques planes, pris comme équivalent. On était donc tenté d'introduire également dans l'équation (21) les lois de la résistance pour couches limites turbulentes. ce qui n'est pas bien difficile. Mais il ne nous a pas semblé possible de donner ici les résultats obtenus avant qu'ils soient confirmés par l'expérience.

\section{Mesure directe des vitesses au voisinage du fond}

La mesure des vitesses intérieures dans la région à écoulement potentiel d'une onde solitaire a déjà été effectuée avec succès à l'aide de photographies de particules colorées; les résultats de ces mesures sont décrits dans l'ouvrage désigné par la réf. 5. Mais ce procédé n'est cependant pas applicable à l'observation des vitesses au voisinage de la paroi pour des raisons suivantes : (a) il n'est pas facile de vérifier les dimensions et la position des gouttelettes et $(b)$ on ne peut considérer comme valables des vitesses mesurées sur une particule qui, en réalité, se trouve au fond. Il a donc fallu imaginer et conștruire un tube de Pitot spécial, à l'effet de mesurer les vitesses à l'intérieur de la couche limite.

Les points suivants ont dû être pris en considération : les hauteurs de vitesse soumises à la mesure étaient exceptionnellement faibles, les 
pressions statiques en un point donné varient au passage de l'onde et ne sont pas répartics hydrostatiquement. Il a donc fallu faire des relevés instantanés, en une même position sous l'onde, de la pression ambiante et de la charge totale. A cet effet, deux tubes ont été reliés à un manomètre différentiel, comme représenté sur la figure 6, pour la mesure respective de ces deux grandeurs. Ils étaient disposés de manière qu'ils débouchent au même niveau en longueur et en alti-

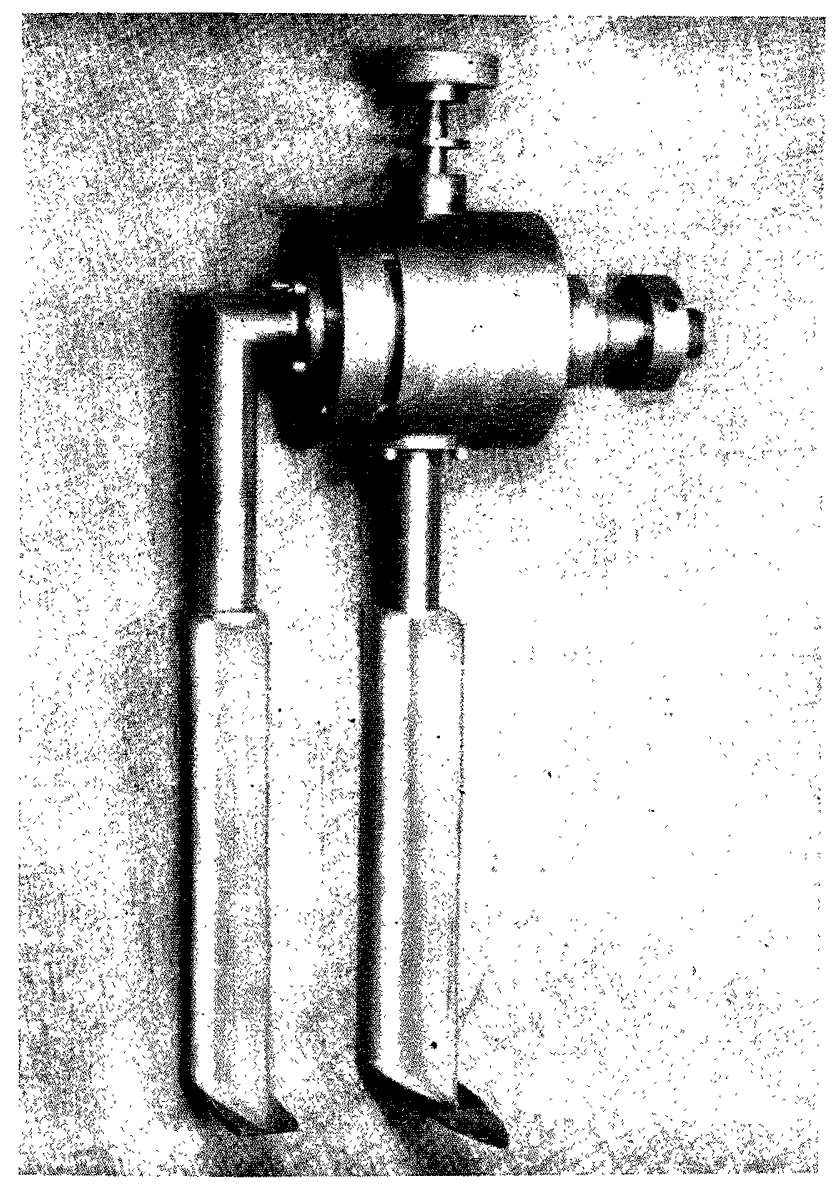

FIG. 6. - Sonde différentielle pour les mesures des fluctuations de vitesse.

Assembled differential Gage for transient Velocity Measurements.

tude, mais avec un léger décalage latéral. La différence des pressions était déterminée au moyen d'un transmetteur de pression du type transformateur ayant son noyau lié au diaphragme.

Le corps de manomètre et les deux tubes qui avaient $22 \mathrm{~mm}$ de diamètre et environ $30 \mathrm{~cm}$ de longueur étaient en laiton. Le diaphragme avait $51 \mathrm{~mm}$ de diamètre et $0,25 \mathrm{~mm}$ d'épaisseur, il était d'une seule pièce avec sa monture annulaire, qui élait en laiton fortement étiré ayant subi un traitement thermique. Des anneaux de section en « 0 » assuraient l'élanchéité en tout point. Les deux sondes sont de même construction, sauf que la sonde statique comporte un profilage à son extrémité amont pour que l'écoulement soit régulier devant son orifice de prise disposé latéralement. Les deux orifices sont de $7 / 8 \times 1 / 32$ in. $(0,7 \mathrm{~mm})$, suffisamment petites, estimait-on, pour empêcher les écoulements secondaires en dedans de l'ouverture, mais assez larges pour ne causer qu'un effet insensible de viscosité sur les mesures de pressions [8]. Des capuchons profilés sont fixés sur l'avant des tubes pour réduire les perturhations possibles de l'écoulement.

Pour empêcher la formation de bulles d'air, on montait chaque fois le manomètre sous l'eau; un chapeau imperméable à l'eau protégeait les conducteurs électriques allant à la bobine du transformateur.

Le transformateur qui enregistre par voie électronique les déplacements du diaphragme, par déplacement de son noyau à l'intérieur de la bobine, est un Linearsyn, modèle SM (de la Control Components Company), à mouvement rectiligne; sa longueur est de $25 \mathrm{~mm}$ et son diamètre extérieur de $19 \mathrm{~mm}$. Les déplacements maximum et minimum du noyau, détectables au moyen d'un dispositif électronique approprié, sont de $0,7 \mathrm{~mm}$ et de $25 \cdot 10^{-7} \mathrm{~mm}$. Le noyau du transformateur a $7 \mathrm{~mm}$ de longueur et $3 \mathrm{~mm}$ de diamètre; il est en alliage nickel-fer et fixé au diaphragme.

Cette liaison est particulièrement délicate, car il ne faut pas qu'elle déforme le diaphragme plan, ou qu'elle altère d'une façon quelconque les caractéristiques de l'appareil. Dans le cas considéré, le noyau était relié à une fine tige de laiton qui portait à son autre bout un mince disque de laiton ayant $3 \mathrm{~mm}$ de diamètre. Ce disque était fixé au diaphragme. Le collage des surfaces était effectué à la soudure "SwifBrush-on »; les éléments assemblés étaient portés dans un four électrique dont on élevait la lempérature jusqu'à $93^{\circ} \mathrm{C}$; on les laissait ensuite refroidir lentement jusqu'à la température de la pièce.

La bobine du transformateur est reliéc à une des extrémités d'une tige finement filetée. En la faisant tourner on détermine le zéro du manomètre de manière que le transformateur agisse constamment sur la partie linéaire de la courbe «puissance obtenue - en fonction du déplacement ». Cette puissance était déterminée à l'aide d'un enregistreur Sanborn série 150.

Les caractéristiques principales du manomètre araient été choisies pour satisfaire aux conditions de fréquence propre, de sensibilité et de forme géométrique requises. On peut définir la sensibilité comme le déplacennent subi par le diaphragme pour une différence de pression unitaire. Dans le cas présent, on cherchait à avoir comme déflection détectable minimum $25 \cdot 10^{-7} \mathrm{~mm}$, obtenue sous la pression diffé- 
rentielle la plus faible (c'est-à-dire sous les hauteurs de vitesse, dans le cas des ondes de faible hauteur), compatible avec la fréquence propre requise.

Il convient que la fréquence propre du manomètre soit aussi élevée que possible relativement à la fréquence à mesurer. Pour l'étude en question, on peut imaginer un «train » d'ondes solitaires qui aurait, dans le cas des profondeurs d'eau utilisées, une fréquence de l'ordre de $1 \mathrm{c} / \mathrm{s}$. Après une étude minutieuse de la fréquence et de la sensibilité, les caractéristiques du manomètre ont été choisies comme suit : $(\alpha)$ fréquence de résonance $4,2 \mathrm{c} / \mathrm{s} ;(b)$ détection minimum correspondant à une vitesse de $1 \mathrm{~cm} / \mathrm{s}$.

Pour avoir une base de référence à laquelle on rapporterait la hauteur de vitesse enregistrée, il a fallu enregistrer le profil de l'onde à chaque enregistrement de vitesse. A cette fin, nous nous sommes servis d'un lecteur de profil, consistant en un fil métallique émaillé d'un calibre de $0,38 \mathrm{~mm}$ et long de $19 \mathrm{~cm}$, introduit verticalement dans le canal, à environ $12 \mathrm{~cm}$ sur le côté de la sonde différentielle et dans l'axe de l'orifice de la prise dynamique. Le noyau du fil et l'eau contenue dans le bassin se comportent comme les deux plaques d'un condensateur, l'isolant qui entoure le fil représentant le diélectrique. A l'aide d'un circuit électronique approprié, le profil de l'onde peut être enregistré par la variation de capacité qui accompagne les fluctuations du niveau. Les profils ont pu ètre rattachés ainsi aux vitesses mesurées; mais des mesures d'amplitude de l'onde ont également été faites sur des clichés photographiques pris au passage de l'onde sur les sondes.

Aussi bien le manomètre différentiel que le fil profilé ont été étalonnés statiquement, procédé qui semblait devoir suffire pour des ondes de faible amplitude. Toutefois, d'après le résultat final on a pu se rendre compte de l'utilité d'un étalonnage dynamique, surtout dans le cas du fil.

La vitesse a été enregistrée aux niveaux $z$ suivants au-dessus du fond lisse du bassin (en $\mathrm{mm}$ ):

$$
0,63-1,9-3,2-5,7-9,5-15,6-26-39-55 .
$$

Comme il n'était pas possible, avec le procédé de génération d'onde employé, de répéter une onde exactement, on créait quatre à dix ondes pour chaque réglage en altitude du manomètre différentiel. On choisissait ensuite dans chaque groupe une onde ayant une amplitude de $33 \mathrm{~mm}$
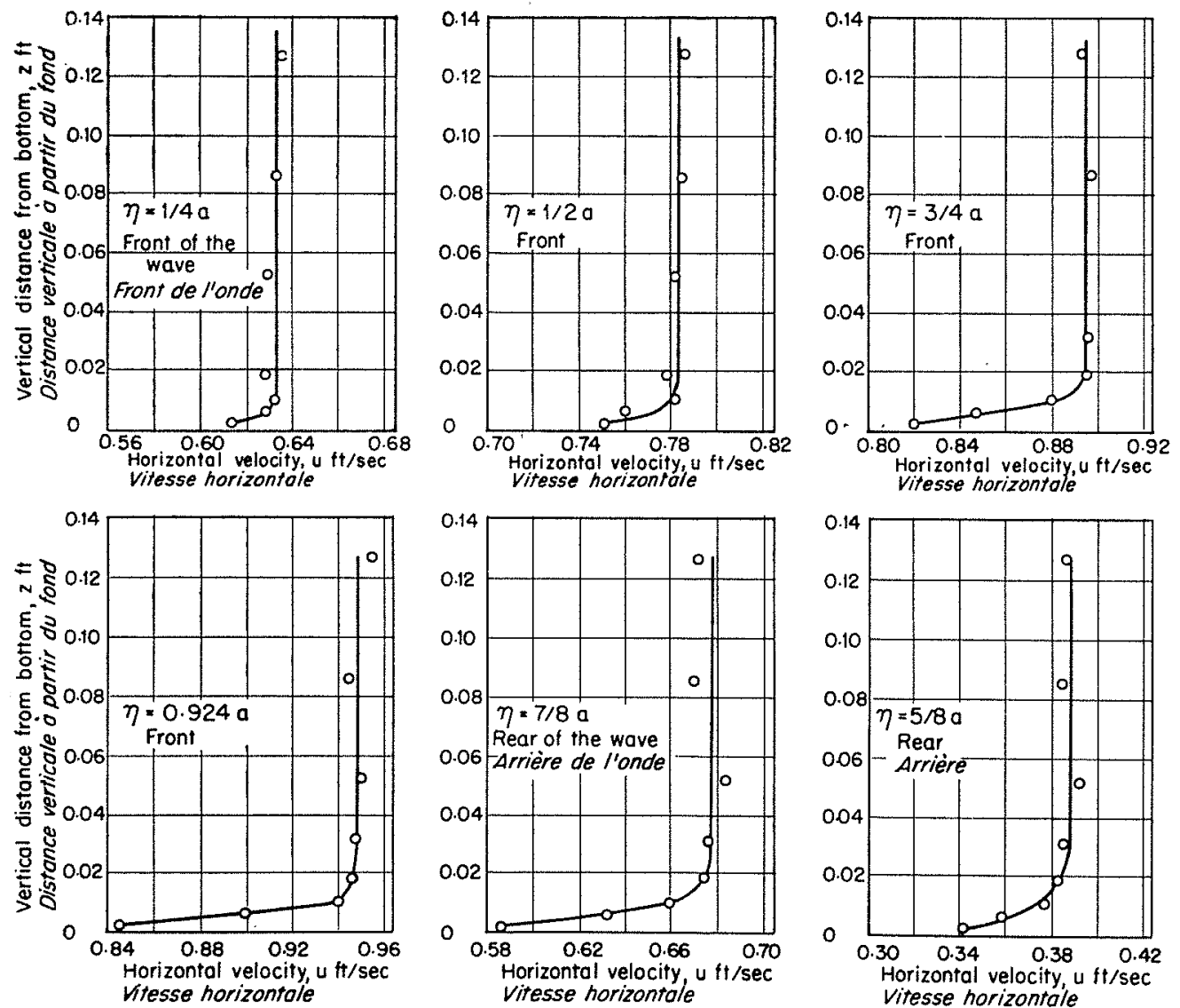

FIG. 7. - Profils de vitcsse dans la couche limite transitoire au passage de londe.

Velocity Profiles in transient Boundary Layer under Wave. 
exactement et on prenait la valeur de la hauteur de vitesse correspondante. La profondeur d'eau au repos a été maintenue à $100 \mathrm{~mm}$. On trouvera dans les ouvrages réf. [7] et [9] de plus amples détails sur la disposition adoptée, l'appareillage et les procédés utilisés.

Sur la figure 7 on voit les profils de vitesse au voisinage du fond calculés pour six valeurs de l'amplitude relative $\eta / a_{0}$ et en différents points le long du profil de l'onde; on les voit également sous forme non-dimensionnelle sur la figure 8 . Contrairement à la théorie, la section à vitesse mesurée maximum se trouvait un peu en amont de la crête en un point où l'élévation de la surface est les 0,924 de l'amplitude de l'onde. On pourrait attribuer ce fait à un retard apparent de l'enregistrement du vrai profil postérieur de l'onde, dû̀ à ce que l'eau adhère légèrement au fil quand la surface s'abaisse.

L'accroissement subi par la couche limite depuis le front jusqu'à l'arrière de l'onde, apparaît clairement sur la figure 8 à travers les variations successives des profils de vitesse. L'épaisseur de cette couche sous la crête est de 3 à $6 \mathrm{~mm}$, selon la façon dont on a défini les limites de la région d'écoulement à potentiel. Selon l'équation (15), l'épaisseur est d'environ $3 \mathrm{~mm}$ pour l'onde étudiée.

On ne saurait trop se fier aux deux profils de vitesse obtenus pour la partie arrière de l'onde. Même si on les décale en arrière pour situer la vitesse maximum sous la crête, une dissymétrie notable subsistera dans les vitesses mesurées en dehors de la couche limite, celles en arrière de la crête semblant trop faibles. Cela peut être dû à la présence d'une petite onde réfléchie depuis les tubes immergés. Il y a éga-

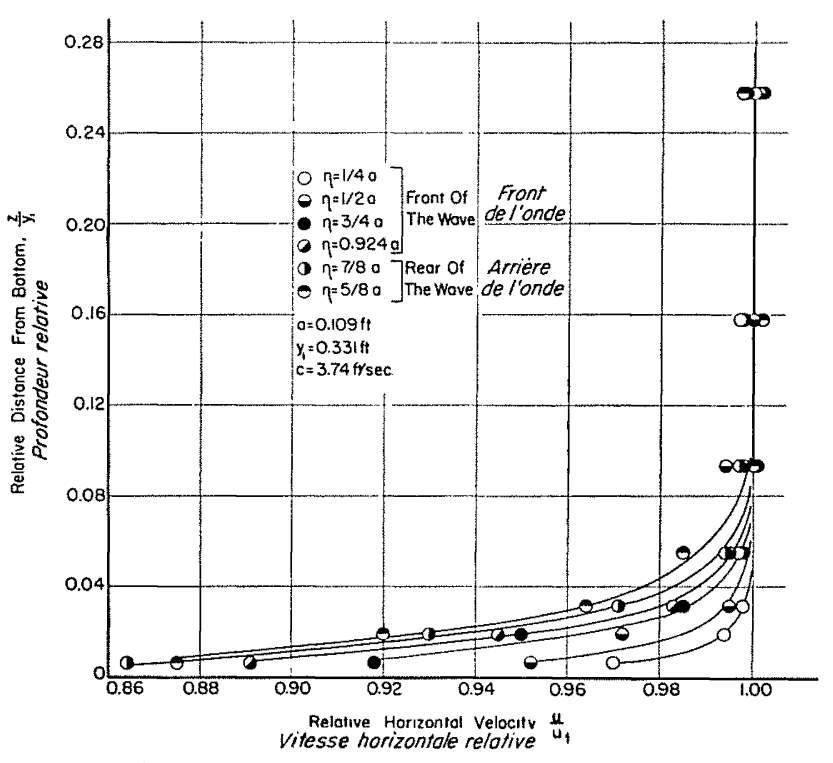

FIG. 8. - Profils des vitesses relatives

dans la couche limite sous une onde solitaire.

Dimensionless Velocity Profiles

in Boundary Layer under solitary Wave.

lement lieu de noter que sous sa forme acluelle, le manomètre ne peut que détecter, et non mesurer, un courant de retour qui suivrait le décollement de la couche limite en arrière de l'onde.

\section{CONCLUSIONS}

Les résultats des expériences peuvent être résumés de la façon suivante :

1. L'affaiblissement de l'amplitude de l'onde et la dissipation d'énergie dans le domaine des conditions étudiées sont dues aux couches limites laminaires transitoires correspondant à des fonds lisses ou rugueux;

2. On a pu arriver à coordonner de façon satisfaisante les résultats expérimentaux avec un nombre de Reynolds déduit uniquement de l'amplitude de l'onde et de la viscosité. Pour établir sa signification physique, on l'a considéré comme équivalent au nombre de Reynolds classique pour plaque plane, dans le cas du frottement pelliculaire laminaire;
3. Les effets de la rugosité sur l'amortissement se sont révélés considérables, parce que les ordres de grandeur de l'épaisseur de la couche limite et des particules constituant la rugosité étaient comparables entre eux. La rugosité relative effective a été définic comme étant le rapport entre le diamètre de la particule, ou du grain, et l'amplitude de l'onde;

4. Une équation empirique a été obtenue donnant le coefficient de résistance global couvrant les cas d'amortissement par fond lisse et par fond rugueux;

5. Des mesures ont été faites de la couche limite transitoire sous une onde solitaire; on a vérifié que ses dimensions correspon- 
daient en gros à la théorie de la plaque plane;

6. D’après le parallèle entre la résistance de plaque plane et l'affaiblissement de l'onde, la valeur de l'amortissement semble être affectée par la présence de couches limites turbulentes aux amplitudes voisines de $18 \mathrm{~cm}$, donc pour des profondeurs au moins égales à $30 \mathrm{~cm}$. Les effets de la rugosité $\mathrm{du}$ fond seront relativement moins accusés pour les ondes de plus fortes amplitudes.
L'étude dont il a été rendu compte ci-dessus fait partie d'un programme de recherches sur les caractéristiques des ondes en eau peu profonde effectuées au Laboratoire d'Hydrodynamique du Massachusetts Institute of Technology. Les mesures de couche limite ont été effectuées par Mr. M. A. RAzA et ont constitué l'objet de sa thèse de Master of sciences. L'exécution des travaux a été entièrement patronné par l'Office of Naval Research du Ministère de la Marine des EtatsUnis.

\section{BIBLIOGRAPHIE - REFERENCES}

1. Boussinese (J.) : « Théorie de l'intumesenee liquide appelée onde solitaire ou de transition $\gg$. Comptes Rendus de l'Académie des Sciences, vol. 72, p. 775,1871

2. Rayleigh (Lord) : «On Waves ». Philosophical Magazine, (5), vol. 1, p. $257,1876$.

3. MaG Gowan (J.) : «On the solitary Wave ». Philosophical Magazine, vol. 32, p. 45, 1891.

4. Munk (W.H.) : "The solitary Wave and its Application to Surf Problems ». Annals of the New York Academy of Science, vol. 51, p. 376, 1949.

5. DaIly (J.W.) and Stephan (S.C.) Jr.: « The solitary Wave ». M.I.T. Hydrodynamics Laboratory, Technical Report $n^{\circ} 8,1952$.
6. Keulegan (G.H.) : " Gradual damping of solitary Waves ». Journal of Research, National Bureau of Standards, vol. 40, p. 487, 1948.

7. Ippen (A.T.), Kulin (G.) and RazA (M.A.) : "Damping Characteristics of the solitary Wave 》. M.I.T. Hydrodynamics Laboratory, Technical Report $\mathrm{n}^{\circ} 16,1955$.

8. Shapiro (A.H.) : « Influence of viscous Effects on Impact Tubes ». Journal of Applied Mechanics, A.S.M.E., vol. 20, $\mathrm{n}^{\circ}$ 2, p. 253, June, 1953.

9. RAZA (M.A.) : " The Measurement of low Velocities in unsteady Flow », M.S. Thesis, Department of Civil and Sanitary Engineering, M.I.T., 1951 (unpublished).

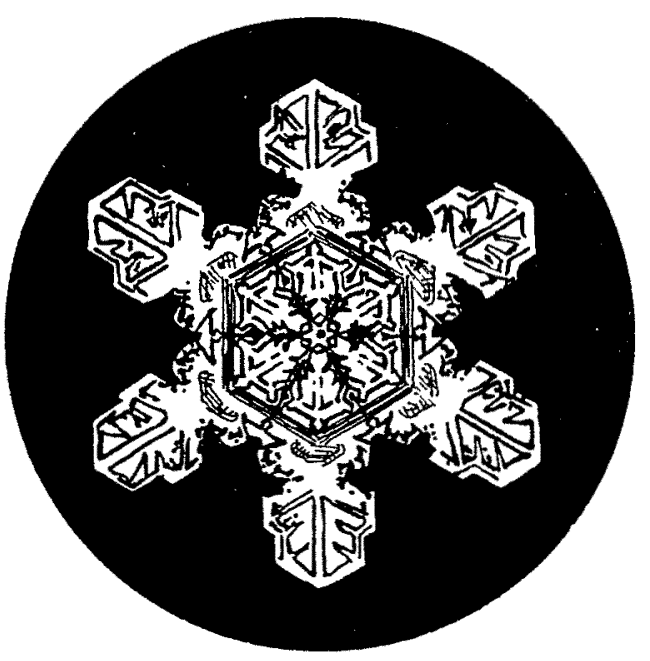




\title{
The effect of boundary resistance on solitary waves
}

\author{
BY \\ ARThur T. IPPEN \\ Professor of Hydraulics \\ Massachusetts Institute of Technology, \\ AND \\ Gershon KULIN \\ Hydraulic ENGineER \\ Nutional Bureau of Standards, Washington, I).C.
}

Texte français, p. 390

\begin{abstract}
This paper reports on the results of an experimental investigation of the attenuation of solitary wave amplitude conducted in the Hydrodynamics Laboratory of the Massachusetts Institute of Technology. The tests were in a lucite channel 32 feet long and 16-1/2 inches wide, with initial water depths ranging approximately from 0.2 feet to 0.4 feet. Some runs were made with the tank bottom artificially roughened with gravel of uniform
\end{abstract}

diameter. Attenualion results are compared with available theory.

In another phase of the study, it was attempted to approach the damping problem by direct measurement of the transient boundary layer near the bottom. A special differential gage for measuring the low transient velocities was designed and the preliminary results obtained with it are described.

\section{INTRODUCTION}

The solitary wave as ordinarily encountered (Figure 1) ${ }^{*}$ ) consists of a single symmetrical crest which is neither preceded nor followed by a trough. A wave of this type travels unchanged in the shape which is given very closely by the Boussinese [1] $\left(^{\star \star}\right)$ equation :

$$
\frac{n}{a_{o}}=\operatorname{sech}^{2}\left[\frac{\sqrt{3}}{2}\left(\frac{a_{n}}{y_{o}}\right)^{3 / 2} \frac{x}{a_{o}}\right]
$$

where $\eta$ is the surface elevation above the original water line at any distance, $x$, from the crest, $a_{o}$ is the maximum value of $\eta$, and $y_{0}$ is the undisturbed depth.

The celerity of the wave form is expressed by the equation of Boussinese and RAYLEIGH [2]:

$$
\frac{\mathrm{C}}{\sqrt{g y_{o}}}=\sqrt{1+\frac{a_{o}}{y_{o}}}
$$

where $\mathrm{C}$ is the celerity and $g$ is the gravitational constant. As a first approximation, therefore, solitary wave celerity can be represented by the

(*) Illustrations are in the French text.

(**) References at the end of the French text (p. ). classical expression for the speed of infinitesimal shallow-water disturbances :

$$
\mathrm{C}={\sqrt{g y_{o}}}_{0}
$$

The internal velocities within the solitary wave are given by the equations of McCowa [3] as non-dimensionalized by MuNK [4].

$$
\begin{aligned}
& \frac{u_{o}}{\mathrm{C}}=\mathrm{N} \frac{1+\cos \mathrm{M} z / y_{0} \times \cosh \mathrm{M} x / y_{0}}{\left(\cos \mathrm{M} z / y_{0}+\cosh \mathrm{M} x / y_{0}\right)^{2}} \\
& \frac{w_{o}}{\mathrm{C}}=\mathrm{N} \frac{\sin \mathrm{M} z / y_{0} \times \sin \mathrm{M} x / y_{0}}{\left(\cos \mathrm{M} z / y_{0}+\cosh \mathrm{M} x / y_{0}\right)^{2}}
\end{aligned}
$$

Here $u_{o}$ and $w_{o}$ are the horizontal and vertical components respectively of the fluid element velocity at elevation $z$ from the bottom and $M$ and $N$ are approximately equal to $\sqrt{3 a_{o} / y_{o}}$ and $2 a_{o} / y_{o}$, respectively. It is seen that for waves of very low amplitude-to-depth ratio the internal velocities are almost entirely horizontal and may be approximated by :

$$
u_{o}=\mathrm{c} \frac{\eta}{y_{0}}
$$


in which case the fluid element velocity is independent of the depth.

The applicability of equations (1), (2), (3a) and $(3 b)$ has been verified by experiments conducted at the Hydrodynamics Laboratory of the Massachusetts Institute of Technology in a lucite tank 32 feet long and 16-1/2 inches wide [5]. The range of initial depths was from 0.2 feet to 0.4 feet.

The volume of a solitary wave per foot of width may be expressed by :

$$
\frac{v}{a_{o}^{2}}=\frac{4}{\sqrt{3}} \frac{1}{\left(a_{o} / y_{o}\right)^{3 / 2}}
$$

and the energy per foot of width by :

$$
\frac{\mathrm{E}}{y a_{o}{ }^{3}}=\frac{3}{2}\left(\frac{4}{\sqrt{3}} \times \frac{1}{\left(a_{o} / y_{o}\right)^{3 / 2}}\right)=\frac{2}{3} \frac{v}{a_{o}^{2}}
$$

where $\gamma$ is the specific weight of water. Equations (5) and (6) are obtained using the profile described by Boussinesq's equation (1). Equation (6) requires that the wave amplitude be extremely small with respect to the depth.

The solitary wave may be regarded as the limiting case of the shallow-water oscillatory wave. The pronounced resemblance of shoaling oscillatory waves to a "train of solitary waves" has led to considerable interest in the solitary wave properties towards predicting the oscillatory wave behavior near the surf zone.

\section{Damping over a Smooth Bottom}

Solitary wave damping can be attributed primarily to the energy loss within a comparatively thin boundary layer which forms along the bottom and the sidewalls of the tank. Outside of this boundary region, the flow remains essentially as described by potential theory.

Fluid element motion within the solitary wave is in the direction of wave motion only, and there is no return flow. A fluid particle which is initially at rest is accelerated in the direction of wave travel under the leading slope of the wave. The particle reaches a maximum velocity under the crest of the wave, following which it decelerates. It comes again to rest when the disturbance, which practically is of finite length, has passed on downstream. A fluid particle near the bottom travels in a straight line under the influence of the wave, while the trajectory of a surface particle is roughly parabolic.

From physical consideration of the particle motion near the boundaries, it can be presumed that there will exist a boundary layer increasing in thickness from front to rear of the wave, since the rear of the wave corresponds to the ends of the particle trajectories. Under the trailing slope of the wave the particles in the boundary layer separation must be recognized.

It is desirable to obtain a Reynolds number descriptive of the internal motion of the wave. In steady-flow flat-plate theory the boundary layer thickness depends upon a Reynolds number which is based on the constant velocity in the potential flow region and on the distance of fluid motion along the plate. A similar Reynolds number can be adopted for use in wave problems if the effect of the unsteadiness of the motion is neglected. This is tantamount to restricting the consideration to very low, long waves-a restriction which is consistent with the range of applicability of equations (2a), (4) and (6).

A physically significant Reynolds number for solitary wave motion may therefore be defined as :

$$
\mathrm{R}_{\varepsilon}=\int \frac{u_{0} d \zeta}{\nu}
$$

where $\zeta$ is the horizontal displacement of fluid particles, $u_{o}$ is the potential flow velocity outside of the boundary layer, and $v$ is the coefficient of kinematic viscosity.

$$
\begin{aligned}
\text { With : } & u_{o}=\frac{d \zeta}{d t} \\
\text { nd : } & \mathrm{C}=\frac{d x}{d t}>>u_{o}
\end{aligned}
$$$$
\text { and : }
$$

equation (7) becomes :

$$
\mathrm{R}_{\tau}=\frac{\mathrm{C}}{\nu y_{0}^{2}} \int_{a}^{\infty} \eta^{2} d x
$$

According to small amplitude theory, the integrand in the equation above is proportional to the wave energy. Using equations $(2 a)$ and (6) and integrating over the entire wave length, the resulting approximate expression for the solitary wave Reynolds number; i.e., the equivalent flat plate Reynolds number is:

$$
R_{\complement}=1.54 \frac{a_{o} \sqrt{g a_{o}}}{\nu}
$$

Assigning average values of $1 / 100,000 \mathrm{ft}^{2} / \mathrm{sec}$ and $32 \mathrm{ft} / \mathrm{sec}^{2}$ to $\nu$ and $g$ respectively, then :

$$
\mathrm{R}_{\complement} \approx 870,000 a_{o}^{3 / 2}
$$

where $a_{o}$ is expressed in feet. 
This Reynolds number, although approximate, is useful for estimating the flow regime within the boundary layer. The highest Reynolds number obtained in the experimental investigation reported here was about 120,000. Comparison with flat plate information indicates that for this value the boundary layer flow can be expected well within the laminar regime.

Again resorting to steady-flow flat-plate theory, the thickness of a laminar layer with parabolic velocity distribution beneath a solitary wave can be expressed as :

$$
\delta_{x}=\frac{5.5 \zeta_{r r}}{\sqrt{\mathrm{R}_{\ell x}}}
$$

Here the subscript « $x \gg$ refers to the cumulative condition from positive infinity to a point $x$ along the wave. In the first approximation the distance of particle motion, $\zeta$, is independent of elevation and is equal to the volume of the intumescence divided by the depth. Then use of equations (5) and (13) gives for the boundary layer thickness at the trailing edge of the solitary wave :

$$
\frac{\delta_{r}=-\infty}{y_{0}}=\frac{12,7}{\sqrt{\mathrm{R}_{\varepsilon}}} \sqrt{\frac{a_{0}}{y_{0}}}
$$

and under the crest :

$$
\delta_{x=0}=\frac{1}{\sqrt{2}} \delta_{r}=-\infty
$$

Equation (14) neglects the effect of the unsteadiness of the motion and does not consider the possibility of flow reversal under the trailing edge of the wave. Such flow reversal is suggested by physical considerations and is in fact indicated by the Keulegan [6] development for boundary shear. Nonetheless, equation (14) provides a direct and easily obtainable estimate of the thickness of the boundary layer beneath a wave of small amplitude.

By equating the energy loss due to viscous damping in the boundary layer to the energy loss corresponding to the decrease in wave amplitude, Keulegan [6] obtained the following expression for solitary wave attenuation:

$$
\begin{aligned}
& \left(\frac{a_{o}}{y_{o}}\right)^{-1 / 4}-\left(\frac{a_{i}}{y_{o}}\right)^{-1 / 4} \\
& \quad=\frac{1}{12}\left(1+\frac{2 y_{n}}{\mathrm{~B}}\right) \sqrt{\frac{\nu}{g^{1 / 2} y_{o}^{3 / 2}}} \times \frac{s}{y_{o}}=\mathrm{K} \frac{s}{y_{o}}
\end{aligned}
$$

Here $B$ is the width of the channel, and $s$ is the distance over which the amplitude is attenuated from $a_{i}$ to $a_{o}$. It is seen that the attenuation coefficient is constant for a fixed depth of water.

The form of equation (16) was verified by experiments [5]. Damping was observed by photographing each wave as it was reflected back and forth from the ends of the 32-foot tank. In these experiments, as in all tests reported in this paper, the solitary waves were generated by the rapid raising of a gate to release a small volume of impounded water which in turn displaced a vertical wall smoothly over a short distance downstream. At each depth several waves of different initial amplitude were observed until extinction. The following average attenuation coefficients were obtained:

\begin{tabular}{c|c|c}
\hline Depth, ft. & Keulegan K & Empirical K \\
\cline { 1 - 2 } 0.2 & 0.000480 & 0.000372 \\
0.3 & 0.000379 & 0.000327 \\
0.4 & 0.000335 & 0.000318
\end{tabular}

The empirical coefficient is seen to depend to a lesser extent upon the depth than the theoretical coefficient.

Differences between theoretical and observed results may be readily anticipated in this case because:

(1) theory, for the sake of workability, employs first-approximation expressions for wave celerity, energy and internal velocities;

(2) the side wall correction term $\left(1+2 y_{0} / \mathrm{B}\right)$ is very approximate for waves of large and moderate height; and

(3) successive reflections, which must be used in order to observe damping in a laboratory channel, produce a certain amount of secondary oscillation, so that the wave never moves through completely quiescent water.

A solitary wave retains essentially its initial volume as it attenuates, although the potentialtheory wave loses in volume as its amplitude diminishes. Experiments [7] have indicated that presumably this volume differential is concentrated near the ends of the wave and that the central portion of the profile of even a severely damped wave is adequately described by potential theory (eq. 1). This difference betwen theoretical and actual volumes has been shown to have a negligible effect on the damping process.

To investigate solitary wave damping without resorting to successive reflections, experimental results were analyzed using only the first pass of the wave down the channel after generation 
The results are shown in Figure 2. Because the distance between the first two observation stations is only twelve feet, the observed amplitude decrease was small and the experimental scatter correspondingly large. However, a pronounced trend is indicated and shows the magnitude of the damping coefficient to vary inversely with the wave height. This result suggested plotting the coefficients against Reynolds number, $R_{\xi}$, as is shown in Figure 3 . While the scatter is seen to be increased, a consistent interrelation seems nevertheless indicated, which is followed up further in the subsequent section.

In the low $a_{0} / y_{o}$ range, where the simplifying assumptions of the Keulegan theory are approximately satisfied, the damping coefficients are somewhat higher in Figure 2 than the values predicted by theory. The reason for this discrepancy is not known, although the pronounced slope of the experimental curve with respect to the horizontal theoretical line mav be explained by comparison of the actual velocities within the wave with the theoretical velocities given by equation (4).

\section{Damping over an Artificially Roughened Bottom}

The damping equation stated in the preceding section (eq. 16) and derived by Keulegan [6] is restricted to the case of energy dissipation in a transient laminar boundary layer under solitary waves passing through smooth channels. Its range of applicability is therefore limited to values of $R_{\varepsilon}$, the equivalent flat-plate Reynolds number, normally considered for laminar boundary layers. A more generally applicable treatment was therefore attempted as a means of correlating damping data obtained with roughened surfaces, which might be useful beyond the laminar damping range for the transition and the turbulent boundary layer damping.

If the local and instantaneous surface resistance is defined as customary by the unit shear:

$$
\tau=c_{f} \frac{\rho u_{o}^{2}}{2}
$$

then:

$$
\frac{d \mathrm{E}}{d t}=\int_{-\infty}^{+\infty} \tau u_{o} d x=\int_{-\infty}^{+\infty} c_{f} \rho \frac{u_{0}{ }^{3}}{2} d x
$$

Substituting equations (1) and (4) and replacing $c_{f}$ by an average coefficient $\mathrm{C}_{f}$ applicable to the entire wave length, the integration in (18) may be performed and:

$$
\frac{d \mathrm{E}}{d t}=\frac{16}{15 \sqrt{3}} \rho \mathrm{C}_{f} g^{3 / 2} a_{n}{ }^{3 / 2}
$$

Differentiating equation (6), a second expression for $d \mathrm{E} / d t$ is obtained:

$\frac{d \mathrm{E}}{d t}=\frac{d \mathrm{E}}{d a} \cdot \frac{d a}{d s} \mathrm{C}=\frac{4}{\sqrt{3}} \rho g^{3 / 2} y_{o}^{2} a_{o}^{1 / 2} \frac{d a}{d s}$

Equating (19) and (20) permits the evaluation of $\mathrm{C}_{f}$ for local values of $d a / d s$ and $a_{0} / y$ from experimental data:

$$
\mathrm{C}_{f}=\frac{15}{4}\left(\frac{a_{o}}{y_{o}}\right)^{-2} \frac{d a}{d s}
$$

It must be anticipated that this general relation will not be integrable unless $\mathrm{C}_{f}$ can be expressed as a function of $R_{\epsilon}$ and of some relative roughness $e / a_{v}$, which ordinarily must be determined experimentally. For the case where $\mathrm{C}_{f}$ is relatively insensitive to changes in either parameter, i.e., for high Reynolds numbers and large $e / a_{0}$ values, equation (21) may be integrated to yield as an ultimate form for the damping equation:

$$
\left(\frac{a_{o}}{y_{0}}\right)^{-1}-\left(\frac{a_{i}}{y_{0}}\right)^{-1}=\frac{4}{15} \mathrm{C}_{f} \frac{s}{y_{0}}
$$

As has been stated previously, the dimension of the waves investigated in the present study were not large enough to find the resistance behavior beyond the equivalent laminar boundary layer range. However, a definite effect of roughness could nevertheless be evaluated and described systematically as shown in the following.

Several series of damping runs were made with the bottom of the wave tank covered with a layer of uniform gravel, which was varied in diameter during the course of the tests from $.0056 \mathrm{ft}$. to $.0198 \mathrm{ft}$. The results of these roughened bottom runs are shown in Figure 4 as curves of $a_{0} / y_{0}$ versus $s / y_{0}$. All curves have been corrected for the effect of smooth side wall resistance in an approximate manner by means of equation (16). For purposes of comparison the curves are grouped in Figure $4 a$ in accordance with depth and in Figure $4 b$ in accordance with the roughness parameter, $e / y_{0}$. It should be noted in Figure $4 b$ that the curves of equal values, $e / y_{0}$, were shifted laterally, where necessary for better comparison, to a starting point of equal initial amplitude to depth ratio, $a_{i} / y_{o}$. Attenuation generally proceeds more rapidly in the case of lower depth with the exception of runs (9) and (2). Run (2), however, was found throughout the analysis inconsistent with the other results and it is very probably in error due to faulty experimental records. The curves in general exhibit predominantly viscous damping with intensities 
clearly dependent on the form resistance of the roughness particles.

The effect of the latter was investigated more in detail by plotting values of $d a / d s$ derived from the primary damping curves versus $a_{0} / y_{0}$ and by computing the resistance coefficients, $\mathrm{C}_{f}$, in accordance with equation (21). The results are given in Figure $5 a$. The values $C_{f}$ generally decrease with increasing Reynolds number, $R_{\varsigma}$, in the same manner as the smooth surface resistance coefficient, $\mathrm{C}_{f}$, defined by the Blasius equation $\mathrm{G}_{f} \vee \mathrm{R}=1.328$ for laminar boundary layers on flat plates. The smooth channel values agree quite well with the Blasius law, thus establishing considerable confidence in the significance of the wave Reynolds number, $R_{\zeta}$, as derived previously. It should be noted that the amplitude alone determines the value of the latter. It was noted also that the vertical shift in $\mathrm{C}_{f}$ values for given values of $\mathrm{R}_{c}$ was essentially governed by the absolute roughness, $e$. Correlations introducing an equivalent plate length or the laminar boundary layer thickness were not successful so far, which may be due to the limited range of the data available. It may be noted here that boundary layer thicknesses computed from equations (14) and (15) are essentially of the order of the roughness particle diameters, $e$, employed in the roughened bottom experiments. However, it was possible to define the relative roughness as $e / a_{0}$ and to derive an empirical expression for $C_{f}$ in terms of $R_{s}$ and $e / a_{0}$ from the data as follows:

$$
\mathrm{C}_{f}=\frac{1.328}{\sqrt{\mathrm{R}_{\mathrm{c}}}}+0.00835 \frac{e}{a_{\mathrm{o}}} \mathrm{R}_{\mathrm{c}}^{1 / 4}
$$

or in the alternate form:

$$
\mathrm{C}_{f}=\frac{1.328}{\sqrt{\mathrm{R}_{\zeta}}}\left(1+0,0063 \frac{e}{a_{\mathrm{o}}} \mathrm{R}_{\zeta}^{3 / 4}\right)
$$

The latter form brings out clearly that the roughness correction which modifies the Blasius constant is only very slightly dependent on the amplitude, $a_{0}$, and depends primarily on the absolute roughness for the range investigated. The value of the constant, .00835, was obtained from the separate plot of Figure $5 \mathrm{~b}$ which shows experimental trends more clearly. With the exception again of the one run for $y_{0}=.20 \mathrm{ft}$ and $e=.0098 \mathrm{ft}$ mentioned before, relatively good agreement exists with the above equations.

It is clear that the waves generated in the tank used in these experiments were not sufficiently large to produce turbulent boundary layers and that the roughness projections are therefore essentially immersed in a region of viscous flow. It was noted earlier that the largest wave Reynolds number obtained was about 120,000 ; a considerably larger value is necessary to establish turbulent action in the boundary layer, notwithstanding the unsteady motion and the roughened bottom which can normally be expected to induce early transition from laminar to turbulent flow. It is estimated that an initial wave amplitude of slightly larger than one foot would be necessary to produce rough-surface damping over an appreciable portion of an attenuation run. This amplitude would require the use of a water depth of at least 1-1/2 feet. A gradual transition from the type of damping indicated by equation (16) to that given by equation (17) must then be expected. Further studies in this area are now in progress.

It is evident that $\mathrm{C}_{f}$ as defined by equations (23) may be introduced into equation (21) for integration and that complete laminar damping curves may then be calculated for smooth and rough channels. For sinooth channels with the Blasius coefficient $C_{f}$, the integration results in the Keulegan damping equation (16) with the only change in the numerical coefficient from $1 / 12$ to $1 / 14$. This essential agreement in the two approaches gives considerable assurance with respect to the physical significance of the equivalent flatplate Reynolds number, $R_{\xi}$. It was tempting, therefore, to introduce also the resistance laws for turbulent boundary layers into equation (21) which can be done without great difficulty. However, it was thought premature to state such results here without experimental confirmation.

\section{Direct Measurement of Near-Bottom Velocities}

The measurement of internal velocities in the potential-flow region of a solitary wave has already been satisfactorily accomplished by the method of photographing dyed droplets and the results have been reported in reference [5]. Such a procedure, however, is not practicable for the observation of velocities in the boundary region because $(a)$ the size and position of the droplets is difficult to control and $(b)$ the measured velocities of a particle which actually rests on the bottom cannot be regarded as reliable. A special pitot tube for the measurement of the velocities in the boundary layer was therefore designed and constructed.

The following points had to be considered: the velocity heads to be measured are unusually small, the static pressures at a given point vary 
as the wave passes and are not hydrostatically distributed. Ambient pressure and total head must therefore be picked up instantaneously and for the same position under the wave. For this reason, two tubes were connected as shown in Figure 6 to a differential pressure cell, one for ambient pressure and one for total head, their position being such as to place their openings at the same longitudinal and vertical location, but slightly displaced laterally. The pressure difference was determined with a transformertype pressure transducer, the core of which was attached to the diaphragm.

The main body of the gage and the two tubes, which are $7 / 8$ inches in diameter and about 12 inches long, are of brass. The diaphragm, 2 inches in diameter and 0.01 inches thick, is constructed monolithically with its mounting ring of heat-treated hard-drawn brass. "O" rings provide watertightness throughout. Both pickup tips are of similar construction, except that the static tip has a streamlined attachment on its upstream edge to insure smooth flow at the static opening, which is located along the side of the tip. Both openings are $7 / 8 \times 1 / 32$ inches, a size which was believed to be small enough to prevent secondary flows within the openings, but large enough to cause no appreciable viscous effect on the detection of pressures [8]. Streamlined shapes are attached along the fronts of the tubes to minimize disturbance to the flow.

To guard against the presence of air bubbles, the gage was assembled under water before each use. For this purpose a watertight cap is provided to cover the electrical leads to the transformer coil.

The transformer, which permits electronic recording of the diaphragm deflection by virtue of the motion of the core within the coil, is a linear motion Linearsyn Model SM (Control Components Company) with length and outside diameter of 1.00 and 0.75 inches, respectively. The maximum and minimum core displacements which can be detected by a suitable electronic setup are 0.03 inches and $1 \times 10^{-7}$ inches, respectively. The transformer core, 0.28 inches long and 0.11 inches in diameter, is of a nickeliron alloy and is attached to the diaphragm.

This is a critical connection, since it is important that it be made in such a manner as not to warp the plane of the diaphragm or otherwise alter its characteristics. In this case the core was connected to a thin brass rod, on the other end of which was attached a thin brass disc $1 / 8$ inches in diameter. This disc was in turn attached to the diaphragm. "Swif Brush-on" solder was applied to the surfaces to be ioined. The parts, clamped together, were placed in an electric oven, the temperature of which was raised to $200^{\circ} \mathrm{F}$ and were then allowed to cool gradually to room temperature.

The transformer coil is attached to one end of a finely threaded shaft. Rotation of this shaft permits the gage to be zeroed in such a manner that the transformer always acts on the linear portion of the output-displacement curve. The output of the transformer was determined with a Sanborn Recorder, Series 150 .

The principal gage dimensions were chosen with regard for the requirements of $(a)$ natural frequency (b) sensitivity and (c) geometry. Sensitivity may be defined as the diaphragm deflection per unit pressure differential. In this case it was desirable to have the minimum detectable deflection, $1 \times 10^{-7}$ inches. This should occur under as small a differential pressure; i.e., velocity head in the case of low waves, as is consistent with the required natural frequency.

It is desirable to have the natural frequency of the gage as high as possible with respect to the frequency to be measured. For the purpose of this investigation, a "train" of solitary waves can be imagined, which, at the water depths employed in these experiments, would have a frequency of the order of 1 cps. A detailed analysis of the gage dimensions with respect to adequate frequency and sensitivity resulted in the following characteristics: $(a)$ the resonant frequency is $4.2 \mathrm{cps}$, $(b)$ the minimum detectable diaphragm deflection corresponds to a velocity of .035 feet per second.

In order to reference the velocity-head record obtained with the gage, it was necessary to obtain a record of the wave profile simultaneously with each velocity record. This was accomplished with a profile gage consisting of a 7-1/2 inch length of 0.015 inch enameled wire which is immersed vertically in the tank about $4-1 / 2$ in. laterally from the differential gage and in line with the dynamic opening. The core of the wire and the water in the tank act as plates of a capacitor, while the wire insulation acts as a dielectric. The change in capacitance associated with the rise and fall of the water surface permits the recording of the wave profile with an appropriate electronic circuit. Although the wave profiles were correlated in this manner with the measured velocities, wave amplitudes were also scaled from photographs taken as the waves passed the gages.

Both the differential gage and the profile wire were calibrated statically, a procedure which was felt to be satisfactory for waves of low amplitude. The final results indicated, however, that a dynamic calibration would have been desirable, particularly in the case of the profile gage. 
Velocity head records were obtained for the following distances $z$ above the smooth bottom of the tank, in feet: $0.0021,0.0063,0.0105$, $0.0188,0.0313,0.0522,0,0855,0.1272$ and 0.1811 . Because exact duplication of waves is not possible with the method of generation employed in this work, for each elevation setting of the differential gage from four to ten waves were generated. From each group a wave was then selected for which the amplitude was exactly 0.109 feet and the velocity head record for this wave was used. The undisturbed water depth was maintained at 0.331 feet. Additional details of design, construction and operating procedure are available in references [7] and [9].

The near-bottom velocity profiles computed for six values of relative amplitude $\eta / a_{0}$ along the length of the wave are shown in Figure 7 and again in dimensionless form in Figure 8 . The section of maximum measured velocity, contrary to theory, occured slightly ahead of the crest at a point where the surface elevation is 0.924 of the wave amplitude. This behavior may reflect an apparent lag in the recording of the true rear profile of the wave due to the tendency of water to adhere slightly to the wire as the surface falls.

The growth of a boundary layer from front to rear of the wave is clearly shown in Figure 8 by the successive changes of the velocity profiles. The boundary layer thickness under the crest is from 0.01 to 0.02 feet, depending upon the manner in which the limits of the potential flow region are designated. The thickness according to equation (15) is approximately 0.01 feet for the wave investigated.

The two velocity profiles obtained for the rear portion of the wave cannot be considered too reliable. Even if the measured profiles are shifted rearward to place the maximum velocity under the crest, a considerable asymmetry remains in the measured velocities outside of the boundary layer, those behind the crest apparently being too low. This behavior could be the result of a small wave reflected from the submerged tubes. It should also be pointed out that the gage in its present form will only detect, but cannot measure, any reversal of flow which may be associated with boundary layer separation beyond the wave.

\section{CONCLUSIONS}

The experimental findings may be summarized by the following statements :

1. Wave amplitude attenuation and energy dissipation for the range of conditions investigated are due to transient laminar boundary layers for smooth and roughened channel bottoms.

2. Satisfactory correlation of the experimental data was achieved on the basis of a Reynolds number defined by wave amplitude and viscosity only. Its physical significance was established as equivalent to the normal flat-plate Reynolds number for laminar skin friction.

3. The effect of roughness on the damping was found considerable due to the comparable order of magnitude of boundary layer thickness and roughness particle diameter. The effective relative roughness was defined as the ratio of particle or grain diameter to the wave amplitude.

4. An empirical equation was obtained for a general resistance coefficient for smooth and roughened bottom damping.
5. The transient boundary layer under a solitary wave has been measured and its magnitude essentially verified in terms of flat-plate concepts.

6. On the basis of correlation of flat-plate resistance and wave attenuation, the range of damping is expected to be affected by turbulent boundary layers approximately at amplitudes of $.60 \mathrm{ft}$. and thus for depths not less than one foot. Rough bottom efrects will be less pronounced relatively for waves of higher amplitudes.

\section{Acknowledgments}

The study reviewed above forms a part of a continuing program on shoaling wave characteristics at the Hydrodynamics Laboratory of the Massachusetts Institute of Technology. The boundary layer measurements were carried out by Mr. M. A. RAZA as thesis research for the degree of Master of Science. The project was supported throughout by the Office of Naval Research of the U.S. Navy Department. 